\title{
Time consistent fiscal policies in a Ramsey economy ${ }^{1}$
}

\author{
Roberto Cellini $^{2}$ and Luca Lambertini ${ }^{3}$
}

October 5, 2004

${ }^{1}$ We thank George Leitmann, Riccardo Rovelli and Biagio Speciale for helpul comments. The usual disclaimer applies.

${ }^{2}$ Dipartimento di Economia e Metodi Quantitativi, Corso Italia 55, 95129 Catania, Italy; phone +39095375344 , fax +39095370574 , e-mail: cellini@unict.it.

${ }^{3}$ Dipartimento di Scienze Economiche, Strada Maggiore 45, 40125 Bologna, Italy; phone +390512092600 , fax +390512092664 , e-mail: lamberti@spbo.unibo.it. ENCORE, Faculty of Economics \& Econometrics, University of Amsterdam, Roetersstraat 11, WB1018 Amsterdam, The Netherlands. 


\begin{abstract}
This paper revisits a well-known case of optimal fiscal policy in a Ramsey model where consumer utility is defined over consumption and public goods. We show that normalising the size of the population to one eliminates the scope for active policy-making since the decentralised equilibrium coincides with social planning. Then, we modify the model to allow for a population of $N>1$ agents, whereby restoring the role of the government as a policymaker. Both in the Stackelberg case and in the decentralised game, we prove that optimal fiscal policy and consumption are not only time consistent but also subgame perfect.
\end{abstract}

J.E.L. Classification: C61, C73, E61, E62

Keywords: differential games, optimal control, fiscal policy 


\section{Introduction}

As is known from Simaan and Takayama (1973a,b) and Kydland (1977), the open-loop solution of a Stackelberg differential game is generally affected by time inconsistency. This has given rise to a wide stream of literature on time (in)consistency and credibility of optimal fiscal and monetary policy, starting with Kydland and Prescott (1977) and Calvo (1978), and continuing with Barro and Gordon (1983a,b) and Lucas and Stokey (1983), to mention only a few. ${ }^{1}$ The issue of time inconsistency lies entirely in the temptation for the government or the central bank to renege initial (open-loop) plans at any intermediate date and re-optimise their policy instruments given the state of the economy at that date. Of course, the availability of a reliable commitment technology would make the resulting open-loop policy plans time consistent. The question is then, whether we may think that such a commitment does indeed exist.

A way out of the time inconsistency problem is studied by Cohen and Michel (1988), and - technically speaking - consists in finding the policy that solves the Bellman equation of the policy maker, through the value function approach. The resulting feedback policy rule is surely strongly time consistent. $^{2}$ Yet, one may argue that the amount of information needed to derive

\footnotetext{
${ }^{1}$ This line of research has been constantly pursued along several directions, up to a recent contribution illustrating the time consistency of the Friedman rule of monetary policy (Alvarez et al., 2004). Other relevant applications concern the interplay between economic policy and elections. For exhaustive overviews, see Persson and Tabellini (1990, 2000).

${ }^{2}$ The literature on differential games distinguishes "weak" from "strong" time consistency: in the former case, the Nash (or Stackelberg) equilibrium is not sugbame perfect, while in the latter it is so. As an intuitive proof, consider a game spanning over time running from 0 to $T$ and then consider the game over the sub-interval $(t, T)$, with $t>0$ : in general, players' optimal choices in the latter setting do not change with respect to the entire game, only if the initial conditions at time $t$ coincide with the state of the system
} 
the feedback Stackelberg equilibrium represents too much of a requirement for the policy maker, who might well be unable to collect and/or use it appropriately. If one accepts this view, then time inconsistency can be considered as the realistic consequence of the fact that policy makers are in some sense forced to adopt simple (open-loop) solutions as they cannot design feedback ones.

However, under some specific conditions, open-loop Stackelberg equilibria can indeed be not only time consistent, but also subgame perfect. This is indeed the case when (i) the leader's control variable(s) does not affect the follower's co-state variable(s), and therefore the leader cannot manipulate the follower's objective function at will, and (ii) feedback effects are absent, in the sense that all controls variable are independent of the state(s). Condition (i) is necessary and sufficient for time consistency, but only necessary for subgame perfection; condition (ii) is necessary but not sufficient to ensure subgame perfection. When holding together, (i-ii) yield subgame perfect open-loop Stackelberg equilibria (see Dockner et al., 2000, especially ch. 5; and Cellini et al., 2004).

The present paper revisits a case of time consistent fiscal policy rule, recently proposed by Karp and Lee (2003) relying on previous research by Xie (1997). As a first step, we show that this setup satisfies sufficient conditions for the resulting optimal fiscal policy to be not only time consistent but also subgame perfect, since (i) the optimal policy is stationary and (ii) the reached at time $t$ in the whole game spanning over $(0, T)$. This invariance in optimal plans denotes weak time consistency. If the plan invariance holds irrespective of the state at time $t$, then time consistency is strong. In the case of sequential moves, i.e., in Stackelberg games, the open-loop equilibrium is in general neither weakly nor strongly time consistent, as at any date the leader finds in general optimal to change its plan. Thus, the time consistency we are dealing in this setting, is the strong time consistency, which entails of course the weak time consistency. For further details see Dockner et al. (2000) and Cellini et al. (2004). 
consumption plan chosen by the representative consumer is unaffected by the state variable at all times during the game.

In addition to that, the setting we investigate allows us to tackle a further issue that, to the best of our knowledge, has been neglected so far. Xie (1997) and Karp and Lee (2003) illustrate an open-loop Stackelberg differential game where the policy maker is the leader and a population of atomistic (i.e., non strategic) consumers are the followers. The economy is a Ramsey one, and fiscal policy is aimed at collecting the resources which are needed to produce a public good. Consumers are taken to be rational but myopic, in the sense that they are aware of the negative effect exerted by taxation on the capital accumulation process, while they take the amount of the public good as given, in that each individual contribution to it is negligible.

As usual in this kind of analysis, the representative consumer assumption is coupled with the assumption of a constant returns to scale technology, so that the production function as well as capital and consumption can be expressed in per capita terms to ease the exposition. However, if this normalisation translates into the assumption that the representative consumer summarises in himself the entire economy, then there seems to appear a delicate problem as to the scope for active policy-making in this model. This issue can be explained as follows. In Xie (1997) and Karp and Lee (2003), the normalisation entails that the amount of the public good available to the entire economy indeed coincides with the individual contribution imputable to the taxation of the representative consumer. If this is the case, then the decentralised equilibrium must necessarily coincide with social planning, which by definition yields the fist best allocation. As a consequence, one cannot expect the Stackelberg model with the policy maker as the leader to perform better than the decentralised equilibrium. The obvious reason for this result is that in such a case the prisoners' dilemma behind the private provision of a public good is simply not there. After proving this, we proceed 
to extend the analysis to the case where the representative agent is one out of $N>1$ symmetric agents operating in the economy, and the amount of public good available to each of them is indeed the sum of all individual contributions. In this setting, the decentralised equilibrium is worse than social planning because of the underlying free-riding incentive, and the inefficiency of the decentralised equilibrium clearly grows larger as the number of agents increases. This restores the scope for an active role of the policy maker in correcting such allocative distortion, i.e., it allows us to illustrate a proper game between the policy maker and the population of atomistic agents, still preserving the desirable time consistency property of the open-loop Stackelberg equilibrium.

The remainder of the paper is structured is as follows. In sections 2 and 3, following Xie (1997), Dockner et al. (2000) and Karp and Lee (2003, section 5 ), we illustrate a case of subgame perfect fiscal policy, relying on the openloop Stackelberg differential game where the policy maker is the leader and a population of atomistic consumers are the followers. In the available literature, this framework is usually considered under the normalization of the population size to one (i.e., under the single agent assumption). We show that this approach leads to the conclusion that the differential game is in fact equivalent to a straightforward optimal control model. In section 4, we extend the model to account for the existence of a population of consumer, i.e., abandoning the normalisation usually associated with the representative agent. This allows us to illustrate a proper game between the policy maker and the population of private agents, still preserving the desirable properties of subgame perfection (and therefore time consistency) of the open-loop Stackelberg equilibrium. In section 5, we present a model in which no policy action takes place: this setting allows us to study the properties of the allocation associated to a decentralized economy in which strategic interaction exists only among private agents. Finally (section 6), we compare the 
allocations across the different regimes, focussing on the implications of the usual single-agent normalization, and the role of public goods. Concluding remarks are gathered in section 7 .

\section{Xie's and Karp-Lee's models revisited}

A competitive economy, existing over $t \in[0, \infty)$, is summarised by a representative agent whose instantaneous utility function is defined as follows:

$$
U(t)=\ln c(t)+\ln g(t)
$$

where $c(t)$ is consumption and $g(t)$ is public expenditure at time $t$. The consumer accumulates productive capital $k(t)$ in order to produce output according to the production function

$$
y(t)=A k(t), \quad A>0
$$

and the government chooses a tax rate $\theta(t) \in[0,1]$ in order to raise the funds needed to finance the public expenditure, $g(t)=\theta(t) y(t)$ at any $t \in[0, \infty)$. Therefore, capital (the state variable) accumulates according to the following Ramsey dynamics:

$$
\dot{k}=A[1-\theta(t)] k(t)-c(t) .
$$

The consumer chooses consumption in order to maximise:

$$
\int_{0}^{\infty} e^{-\rho t}\{\ln c(t)+\ln g(t)\} d t
$$

where $\rho \in[0, \infty)$ is the discount rate, subject to (3) and to the initial condition $k(0)=k_{0}>0$.

The model assumes that the government plays as a Stackelberg leader, while the agent plays as a Stackelberg follower, that is, taking the choice of the leader, $\theta(t)$ as given. 
Therefore, the follower's Hamiltonian is:

$$
\mathcal{H}_{F}=e^{-\rho t}\left\{\ln c(t)+\ln g(t)+\lambda_{F}(t)[A(1-\theta(t)) k(t)-c(t)]\right\}
$$

where $\lambda_{F}(t)=\mu_{F}(t) e^{\rho t}$ is the co-state variable (evaluated at time $t$ ) associated with the state variable $k(t)$. At this stage, it is worth noting that taxation explicitly appears only in the kinematics of capital, while the argument of the instantaneous utility is expressed as public expenditure. This means that the atomistic consumer is directly affected by the tax rate only when deciding how much to accumulate.

The first order conditions (FOCs) are: ${ }^{3}$

$$
\begin{gathered}
\frac{\partial \mathcal{H}_{F}}{\partial c(t)}=\frac{1}{c(t)}-\lambda_{F}(t)=0 \\
-\frac{\partial \mathcal{H}_{F}}{\partial k(t)}=\dot{\lambda}_{F}(t)-\rho \lambda_{F}(t) \Leftrightarrow \dot{\lambda}_{F}(t)=\lambda_{F}(t)[\rho-A(1-\theta(t))]
\end{gathered}
$$

and the transversality condition is:

$$
\lim _{t \rightarrow \infty} e^{-\rho t} \lambda_{F}(t) k(t)=0
$$

Note that the adjoint equation (7) does not feature the feedback effect

$$
\frac{\partial \mathcal{H}_{F}}{\partial \theta(t)} \cdot \frac{\partial \theta(t)}{\partial k(t)}
$$

exerted by state $k(t)$ on control $\theta(t)$, i.e., the present solution is indeed of the open-loop type.

From (6), we obtain:

$$
c(t)=\frac{1}{\lambda_{F}(t)}
$$

This entails that, at any instant $t$, the co-state variable of the follower is independent of the tax rate $\theta(t)$. Therefore, the game is uncontrollable by the

\footnotetext{
${ }^{3}$ The indication of exponential discounting is omitted for the sake of brevity.
} 
leader (Xie, 1997), and the open-loop Stackelberg solution is time consistent (Karp and Lee, 2003, Lemma 1, p. 356).

It is possible to further characterize the follower's problem. Substituting (10) into (3) and multiplying all terms by $\lambda_{F}(t)$ we obtain

$$
\lambda_{F}(t) \dot{k}(t)=A(1-\theta(t)) \lambda_{F}(t) k(t)-1
$$

Consider now (7) and multiply all its terms by $k(t)$ :

$$
k(t) \dot{\lambda}_{F}(t)=k(t) \lambda_{F}(t)[\rho-A(1-\theta(t))]
$$

The sum of (11) and (12) gives:

$$
k(t) \dot{\lambda}_{F}(t)+\lambda_{F}(t) \dot{k}(t)=d\left[k(t) \lambda_{F}(t)\right] / d t=\lambda_{F}(t) k(t) \rho-1 .
$$

The solution of the differential equation (13) is

$$
\left[k(t) \lambda_{F}(t)\right]=1 / \rho+Q e^{\rho t}
$$

where $Q$ is an integration constant. In order to satisfy the transversality condition (8), it is necessary to set $Q=0$. Thus, $\left[k(t) \lambda_{F}(t)\right]=1 / \rho$, and considering (10), we have:

$$
c(t)=\rho k(t)
$$

Thus, the dynamic constraint can be rewritten as

$$
\dot{k}=A[1-\theta(t)] k(t)-\rho k(t)
$$

whose solution is

$$
k(t)=k_{0} \exp \left[\int_{0}^{t}[(1-\theta(s)) A-\rho] d s\right]
$$

and hence

$$
c(t)=\rho k_{0} \exp \left[\int_{0}^{t}[(1-\theta(s)) A-\rho] d s\right]
$$


The above dynamic equations describes the choices pertaining to the consumer when he's taking the follower's role.

In order to characterise the optimal fiscal policy, we have to build the policy maker's Hamiltonian. The government chooses $\theta(t)$ in the leader's position, so as to maximise the discounted utility of the representative consumer, under the constraints (3) and (7), and under the follower's optimal consumption decision $c(t)=1 / \lambda_{F}(t)$, which is accounted for by the leader. The corresponding Hamiltonian function is

$$
\begin{gathered}
\mathcal{H}_{L}=e^{-\rho t}\left\{\ln \left[1 / \lambda_{F}(t)\right]+\ln [A k(t) \theta(t)]+\lambda_{L}(t)[A(1-\theta(t)) k(t)+\right. \\
\left.\left.-1 / \lambda_{F}(t)\right]+\varphi_{L}(t) \lambda_{F}(t)[\rho-A(1-\theta)]\right\}
\end{gathered}
$$

where $\lambda_{L}(t)=\mu_{L}(t) e^{\rho t}$ and $\varphi_{L}(t)=\varpi_{L}(t) e^{\rho t}$ are the co-state variables (evaluated at time $t$ ) associated with the state variable $k(t)$ and the follower's co-state variable $\lambda_{F}(t)$, respectively.

The FOCs of the leader are $:^{4}$

$$
\begin{gathered}
\frac{\partial \mathcal{H}_{L}}{\partial \theta(t)}=\frac{1}{\theta(t)}-A\left(\lambda_{L}(t) k(t)+\varphi_{L}(t) \lambda_{F}(t)\right)=0 \\
-\frac{\partial \mathcal{H}_{L}}{\partial k(t)}=\dot{\lambda}_{L}(t)-\rho \lambda_{L}(t) \\
-\frac{\partial \mathcal{H}_{L}}{\partial \lambda_{F}(t)}=\dot{\varphi}_{L}(t)-\rho \varphi_{L}(t)
\end{gathered}
$$

At this stage, it is worth noting again that neither (21) nor (22) contain feedback effects due to the fact that the representative consumer's FOC, equation (10), does not contain the state variable $k(t)$, so that the government's FOCs are defined for the attainment of the open-loop solution.

Equations (20-22) imply, respectively:

$$
\theta(t)=1 /\left[A \lambda_{L}(t) k(t)-\varphi_{L}(t) \lambda_{F}(t)\right]
$$

\footnotetext{
${ }^{4}$ Again, we omit the indication of time and exponential discounting for the sake of brevity.
} 


$$
\begin{gathered}
\dot{\lambda}_{L}(t)=\lambda_{L}(t)[\rho-A(1-\theta(t))]-\frac{1}{k(t)} \\
\dot{\varphi}_{L}(t)=\frac{\lambda_{F}^{2} A(1-\theta(t)) \varphi_{L}(t)+\lambda_{F}(t)-\lambda_{L}(t)}{\lambda_{F}^{2}(t)}
\end{gathered}
$$

Now substitute $\lambda_{F}(t)=1 /(\rho k(t))$ into the state dynamics, to obtain

$$
\dot{k}=A[1-\theta(t)] k(t)-\rho k(t),
$$

that is

$$
\lambda_{L}(t) \dot{k(t)}=\lambda_{L}(t) A[1-\theta(t)] k(t)-c(t) \lambda_{L}(t) .
$$

By multiplying all terms of (24) by $k(t)$ we obtain:

$$
k(t) \dot{\lambda}_{L}(t)=k(t) \lambda_{L}(t)[\rho-A(1-\theta(t))]-1 .
$$

Summing up (27) and (28) yields

$$
\lambda_{L}(t) \dot{k(t)}+k(t) \dot{\lambda}_{L}(t)=d\left[\lambda_{L}(t) k(t)\right] / d t=-1
$$

This differential equation has the simple solution:

$$
\left[\lambda_{L}(t) k(t)\right]=G-t
$$

where $G$ is an integration constant. Hence,

$$
\lambda_{L}(t)=(G-t) / k(t)=(G-t) \lambda_{F}(t) \rho .
$$

Substituting (31) into (25) and multiplying all its terms by $\lambda_{F}(t)$ we obtain

$$
\lambda_{F}(t) \dot{\varphi}_{L}(t)=\lambda_{F}(t) A(1-\theta(t)) \varphi_{L}(t)+1-(G-t) \rho .
$$

Now consider the dynamic constraint regarding $\lambda_{F}(t)$ and multiply all its terms by $\varphi_{L}(t)$, so to obtain

$$
\varphi_{L}(t) \dot{\lambda}_{F}(t)=\varphi_{L}(t) \lambda_{F}(t)[\rho-A(1-\theta(t))]
$$


Combining (32) and (33) yields

$$
d\left[\lambda_{F}(t) \varphi_{L}(t)\right] / d t=\lambda_{F}(t) \varphi_{L}(t) \rho+1-(G-t) \rho .
$$

whose solution is:

$$
\left[\lambda_{F}(t) \varphi_{L}(t)\right]=Q e^{\rho t}+G-t-(2 / \rho)
$$

where $Q$ and $G$ are integration constants. It is now possible to rewrite condition (23) as follows:

$$
\theta(t)=\frac{1}{A\left[G-t-Q e^{\rho t}-(G-t)+(2 / \rho)\right]}=\frac{\rho}{A\left(2-\rho Q e^{\rho t}\right)}
$$

The condition $\theta(t) \geq 0$ for any $t$ (including the limit case where $t$ tends to infinity) leads to set $Q=0$, and provides the solution

$$
\theta(t)=\frac{\rho}{2 A}
$$

Notice that the tax rate representing the solution of the optimal problem of the policy maker is constant over time. In particular, it is independent of $k(t)$ for all $t \in[0, \infty)$. This, jointly with the fact that $(10)$ is also independent of the capital endowment at any instant, makes the optimal tax rate chosen by the leader not only time consistent but also subgame perfect:

Proposition 1 The optimal taxation policy set by the government in the open-loop Stackelberg game is part of a subgame perfect equilibrium.

To this regard, it is worth stressing that the additive separability of the Hamiltonian function w.r.t. state and control variables is sufficient but by no means necessary to ensure the subgame perfection of the open-loop Stackelberg outcome (see Cellini et al., 2004). This has the consequence that the optimal policy defined by (37) is stationary (see Karp and Lee, 2003, p. 357). 
The above exposition summarises the main argument contained in Xie's as well as Karp and Lee's analysis, to prove the subgame perfection of the optimal taxation policy in the case of a logarithmic utility function.

Now, taking into account the leader's optimal behaviour, we can write the time path of the choice variable set by the private agent as well as the time path of the capital stock: plugging (37) into (17) and (18) we have, respectively:

$$
\begin{gathered}
k(t)=k_{0} \exp \left[\int_{0}^{t}[(1-\theta(s)) A-\rho] d s\right]=k_{0} \exp \left[\frac{2 A-3 \rho}{2} \cdot t\right] \\
c(t)=\rho k_{0} \exp \left[\int_{0}^{t}[(1-\theta(s)) A-\rho] d s\right]=\rho k_{0} \exp \left[\frac{2 A-3 \rho}{2} \cdot t\right]
\end{gathered}
$$

Note that $k(t)$ and $c(t)$ monotonically increase over time, provided that $A>$ $3 \rho / 2$. Public spending evolves according to the following equation

$$
g(t)=\theta A k(t)=\rho k(t) / 2=\frac{1}{2} \rho k_{0} \exp \left[\frac{2 A-3 \rho}{2} \cdot t\right]
$$

Capital, consumption and the public good entails the condition $A>3 \rho / 2$. In the next section, we re-examine the issue of optimal taxation in an optimal control setting.

\section{The optimal control problem of the repre- sentative agent}

The aim of this section is to highlight that the Stackelberg game is indeed inefficient in terms of its welfare performance, as it introduces strategic interaction in a model that, per se, would be an optimal control problem with a single agent. To do this, we may proceed as follows. We keep the assumption of a single agent, and suppose this representative consumer/producer (or a benevolent planner) chooses both consumption and taxation, using the piece 
of information $g(t)=A k(t) \theta(t)$ everywhere. Thus, he faces the following problem:

$$
\begin{aligned}
\max _{c(t), \theta(t)} & \int_{0}^{\infty} e^{-\rho t}\{\ln c(t)+\ln [A k(t) \theta(t)]\} d t \\
\text { s.t. } & : \quad \dot{k}(t)=(1-\theta(t)) A k(t)-c(t) ; \\
k(0) & =k_{0}>0
\end{aligned}
$$

The corresponding Hamiltonian is:

$$
\mathcal{H}=e^{-\rho t}\{\ln c(t)+\ln [A k(t) \theta(t)]+\lambda(t)[A(1-\theta(t)) k(t)-c(t)]\}
$$

Taking FOCs, we obtain:

$$
\begin{gathered}
\frac{\partial \mathcal{H}}{\partial c(t)}=\frac{1}{c(t)}-\lambda(t)=0 \Leftrightarrow \\
\Leftrightarrow \frac{1}{c(t)}=\lambda(t) \\
\frac{\partial \mathcal{H}}{\partial \theta(t)}=\frac{1}{\theta(t)}-\lambda(t) A k(t)=0 \Leftrightarrow \\
\Leftrightarrow \frac{1}{\lambda(t) A k(t)}=\theta(t) \\
-\frac{\partial \mathcal{H}}{\partial k(t)}=\dot{\lambda}(t)-\rho \lambda(t) \Leftrightarrow \\
\Leftrightarrow \quad \dot{\lambda}(t)=\lambda(t)[\rho-A(1-\theta(t))]-\frac{1}{k(t)}
\end{gathered}
$$

to be considered with the transversality condition

$$
\lim _{t \rightarrow \infty} e^{-\rho t} \lambda(t) k(t)=0
$$

Substituting (44) into the constraint (42) we have $\dot{k}=A[1-\theta(t)] k(t)-$ $1 / \lambda(t)$, i.e.:

$$
\lambda(t) \dot{k}(t)=\lambda(t) A[1-\theta(t)] k(t)-1
$$


Consider now condition (46) and multiply all its term by $k(t)$, so as to obtain

$$
k(t) \dot{\lambda}(t)=k(t) \lambda(t)[\rho-A(1-\theta(t))]-1 .
$$

Summing up (48) and (49), we obtain

$$
\lambda(t) \dot{k}(t)+k(t) \dot{\lambda}(t)=d[\lambda(t) k(t)] d t=\rho k(t) \lambda(t)-2
$$

whose solution is $[\lambda(t) k(t)]=(2 / \rho)+Q e^{\rho t}$, where $Q$ is an integration constant, which has to be set equal to zero in order to fulfill the transversality condition (47). Hence, we obtain $\lambda(t)=2 /[\rho k(t)]$, and, from (44) and (45), by simple substitutions:

$$
\begin{gathered}
c(t)=\frac{\rho k(t)}{2} \\
\theta(t)=\frac{\rho}{2 A}
\end{gathered}
$$

It is immediate to see that the solution of this optimal control problem coincides with the Stackelberg game as concerns the taxation rule, as long as (52) coincides with (37). The intuition behind the coincidence that we have just identified is that the policy maker borrows the objective function of the representative (atomistic) consumer and chooses the optimal policy acting on behalf of such consumer.

However, thanks to simple substitutions we can find the time path of all other relevant variables in the present setting of the command optimum problem, and we can check that they are different with respect to the Stackelberg game. Specifically, in the present optimal control problem, the dynamics of the capital stock is $\dot{k}(t)=A[1-\theta(t)] k(t)-c(t)=(A-\rho) k(t)$, from which it is immediate to derive:

$$
k(t)=k_{0} \exp [(A-\rho) \cdot t]
$$

and hence

$$
c(t)=\frac{\rho}{2} k_{0} \exp [(A-\rho) \cdot t]
$$


while public spending evolves according to

$$
g(t)=\theta A k(t)=\rho k(t) / 2=\frac{1}{2} \rho k_{0} \exp [(A-\rho) \cdot t]
$$

Note that capital, consumption and public good increase over time as long as $A>\rho$. This condition is milder than the one generated by the Stackelberg setting.

\section{Stackelberg game vs command optimum with strategic private agents}

In this section, we study the Stackelberg game and the command optimum allocation, when the private sector is populated by $N>1$ agents. In this case, the usual inefficiency affecting the private provision of a public good is observed, as the economy is populated by a multiplicity of agents, each one being driven by the incentive to free ride upon his mates' contributions.

Examine first the Stackelberg game. Each private agent $i$ (with $i=$ $1, \ldots N)$ chooses his consumption level $c_{i}(t)$, and behaves as a follower with respect to the government (leader) setting the tax policy, i.e., the tax rates $\theta_{i}(t), i=1, \ldots N$, at any $t \in[0, \infty)$.

It is worth stressing that any private agent benefits from the whole amount of the public good (which is non-rival and non-excludable), the amount of public good available at any date $t$ being $G(t)=\sum_{i=1}^{N} g_{i}(t)=$ $\sum_{i=1}^{N} \theta_{i}(t) A k_{i}(t)$. As in the previous sections, all variables are measured in per capita terms, but considering explicitly $N>1$ entails a crucial difference between this and the previous setup, in that $G(t) \neq g_{i}(t)$.

Thus, each private agent $i$ faces the following problem:

$$
\max _{c_{i}(t)} \int_{0}^{\infty} e^{-\rho t}\left\{\ln c_{i}(t)+\ln G(t)\right\} d t
$$




$$
\begin{aligned}
\text { s.t. } & : \quad G(t)=\sum_{h=1}^{N} \theta_{h}(t) A k_{h}(t) \\
\dot{k}_{h}(t)= & A(1-\theta(t)) k_{h}(t)-c_{h}(t) ; \quad(h=1, \ldots N) \\
k_{h}(0)= & k_{h 0}>0 ; \quad(h=1, \ldots N)
\end{aligned}
$$

The corresponding Hamiltonian is:

$$
\begin{gathered}
\mathcal{H}_{F}=e^{-\rho t}\left\{\ln c_{i}(t)+\ln \left[\sum_{h=1}^{N} A \theta_{h}(t) k_{h}(t)\right]\right. \\
\left.+\sum_{h=1}^{N} \lambda_{i h}(t)\left[A(1-\theta(t)) k_{h}(t)-c_{h}(t)\right]\right\}
\end{gathered}
$$

The FOCs are:

$$
\begin{aligned}
& \frac{\partial \mathcal{H}_{F}}{\partial c_{i}(t)}=\frac{1}{c_{i}(t)}-\lambda_{F i i}(t)=0 \\
&-\frac{\partial \mathcal{H}_{F}}{\partial k_{i}(t)}= \dot{\lambda}_{F i i}(t)-\rho \lambda_{F i i}(t) \Leftrightarrow \\
& \Leftrightarrow \dot{\lambda}_{F i i}(t)=\lambda_{F i i}(t)[\rho-A(1-\theta(t))] \\
&-\frac{\partial \mathcal{H}_{F}}{\partial k_{i j}(t)}=\dot{\lambda}_{F i j}(t)-\rho \lambda_{F i j}(t) \Leftrightarrow \\
&\left.\Leftrightarrow \quad \dot{\lambda}_{F i j}(t)=\lambda_{F i j}(t)[\rho-A(1-\theta(t))] \text { for all } j \neq i\right)
\end{aligned}
$$

The problem exhibits separate dynamics concerning the state variables $k_{h}$ $(h=1, \ldots N)$, so that we can set $\lambda_{F i j}(t)=0$ for $j \neq i$.

We assume symmetry across private agents and across tax rates so that $c_{i}(t)=c_{j}(t)=c(t), k_{i}(t)=k_{j}(t)=k(t)$ and $\theta_{i}(t)=\theta_{j}(t)=\theta(t)$ for any $i, j$ at any $t$, so that we have $G(t)=N \theta(t) A k(t)$. As a consequence, also $\lambda_{F i i}(t)=\lambda_{F j j}(t)=\lambda_{F}(t)$ holds.

It is immediate to check that, under symmetry, the solution of the problem is formally identical to the solution of the problem investigated by Xie (1997) 
and Karp and Lee (2003) (see above, section 2), and in particular $c(t)=$ $1 / \lambda_{F}(t)$.

The government, in the position of the Stackelberg leader, faces the following problem (in which the symmetry across private agents has been already taken into account):

$$
\begin{aligned}
\max _{\theta(t)} \int_{0}^{\infty} & e^{-\rho t} N\{\ln c(t)+\ln G(t)\} d t \\
\text { s.t. } & : \quad G(t)=N \theta(t) A k(t) \\
\dot{k}(t) & =A(1-\theta(t)) k(t)-c(t) \\
k(0) & =k_{0}>0 \\
c(t) & =1 / \lambda_{F}(t) \\
\dot{\lambda}_{F}(t) & =\lambda_{F}(t)[\rho-A(1-\theta(t))]
\end{aligned}
$$

The corresponding Hamiltonian function is

$$
\begin{gathered}
\mathcal{H}_{L}=e^{-\rho t}\left\{N \ln \left[1 / \lambda_{F}(t)\right]+N \ln [N A k(t) \theta(t)]+N \lambda_{L}(t) A(1-\theta(t)) k(t)+\right. \\
\left.-1 / \lambda_{F}(t)+N \varphi_{L}(t) \lambda_{F}(t)[\rho-A(1-\theta)]\right\}
\end{gathered}
$$

Apart from the multiplicative term $N$ and a from an additive term $\ln N$ in the objective function, (65) coincides with the problem characterised in section 2. Its complete solution is:

$$
\begin{aligned}
\theta(t) & =\frac{\rho}{2 A} ; \\
k(t) & =k_{0} \exp \left[\frac{2 A-3 \rho}{2} \cdot t\right] ; \\
c(t) & =\rho k_{0} \exp \left[\frac{2 A-3 \rho}{2} \cdot t\right] ; \\
G(t) & =\theta(t) N A k(t)=\frac{\rho}{2} N k_{0} \exp [(A-\rho) \cdot t] .
\end{aligned}
$$

The outcome of the open-loop Stackelberg equilibrium is subgame perfect (and therefore also time consistent), since (61) is independent of $k(t)$ and 
$\theta(t)$, and the optimal tax rate is constant. Hence, the equivalent of Proposition 1 also holds here.

As to the command optimum in the case of a population of $N$ identical private agents, the problem can be written as :

$$
\begin{array}{ll} 
& \left.\max _{c(t), \theta(t)} \int_{0}^{\infty} e^{-\rho t} N\{\ln c(t)+\ln [N A]+\ln k(t)+\ln \theta(t)]\right\} \\
\text { s.t. } & : \quad \dot{k}(t)=(1-\theta(t)) k(t)-c(t) ; \quad k(0)=k_{0}>0
\end{array}
$$

so that it is formally equivalent to the problem we have solved in section 3 , except for the multiplicative factor $N$ and the constant additive term $\ln (N A)$ in the objective function. Its complete solution turns out to be:

$$
\begin{aligned}
\theta(t) & =\frac{\rho}{2 A} ; \\
k(t) & =k_{0} \exp [(A-\rho) \cdot t] \\
c(t) & =\rho k_{0} \exp [(A-\rho) \cdot t] ; \\
G(t) & =\theta(t) N A k(t)=\frac{\rho}{2} N k_{0} \exp [(A-\rho) \cdot t]
\end{aligned}
$$

The conclusion is that the explicit consideration of a multiplicity of strategic private agents, which entails a proper strategic interaction within the private sector (along with the interaction between the private sector and the government) does not undermine the subgame perfection of the optimal fiscal policy.

\section{The decentralized economy}

Suppose that each of $N$ identical private agents choose the level of individual consumption $c_{i}(t)$, and the share $\theta_{i}(t)$ of the individual income devolved to the purchase of a public good, which remains of course non-rival and nonexcludable. Thus, the total amount of public good available at any date $t$ is $G(t)=\sum_{i=1}^{N} \theta_{i}(t) A k_{i}(t)$. 
In this setting, where the government's intervention is assumed away, and each private agent $i$ solves the following problem with respect to the control variables $c_{i}(t)$ and $\theta_{i}(t)$ :

$$
\begin{aligned}
& \max \int_{0}^{\infty} e^{-\rho t}\left\{\ln c_{i}(t)+\ln G(t)\right\} d t \\
\text { s.t. } & : \quad G(t)=\sum_{h=1}^{N} \theta_{h}(t) A k_{h}(t) \\
\dot{k}_{h}(t) & =A(1-\theta(t)) k_{h}(t)-c_{h}(t) ;(h=1, \ldots N) \\
k_{h}(0) & =k_{h 0}>0 ;(h=1, \ldots N)
\end{aligned}
$$

The corresponding Hamiltonian function can be written as

$$
\begin{gathered}
\mathcal{H}_{i}=e^{-\rho t}\left\{\ln c_{i}(t)+\ln \left[A\left(\theta_{i}(t) k_{i}(t)+\sum_{j \neq i} \theta_{j}(t) k_{j}(t)\right)\right]+\right. \\
\left.+\lambda_{i i}(t)\left[A\left(1-\theta_{i}(t)\right) k_{i}(t)-c_{i}(t)\right]+\sum_{j \neq i} \lambda_{i j}(t)\left[A\left(1-\theta_{j}(t)\right) k_{j}(t)-c_{j}(t)\right]\right\}
\end{gathered}
$$

and the FOCs are:

$$
\begin{gathered}
\frac{\partial \mathcal{H}_{i}}{\partial c_{i}(t)}=\frac{1}{c_{i}(t)}-\lambda_{i i}(t)=0 \\
\frac{\partial \mathcal{H}_{i}}{\partial \theta_{i}(t)}=\frac{k_{i}(t)}{k_{i}(t) \theta_{i}(t)+\sum_{j \neq i} k_{j}(t) \theta_{j}(t)}-\lambda_{i i}(t) A k_{i}(t)=0 \\
-\frac{\partial \mathcal{H}_{i}}{\partial k_{i}(t)}=\dot{\lambda}_{i i}(t)-\rho \lambda_{i i}(t) \Leftrightarrow \\
\Leftrightarrow \quad \dot{\lambda}_{i i}(t)=\lambda_{i i}(t)\left[\rho-A\left(1-\theta_{i}(t)\right)\right]-\frac{\theta_{i}(t)}{k_{i}(t) \theta_{i}(t)+\sum_{j \neq i} k_{j}(t) \theta_{j}(t)} \\
-\frac{\partial \mathcal{H}_{i}}{\partial k_{j}(t)}=\dot{\lambda}_{i j}(t)-\rho \lambda_{i j}(t) \Leftrightarrow \\
\Leftrightarrow \quad \dot{\lambda}_{i j}(t)=\lambda_{i j}(t)\left[\rho-A\left(1-\theta_{j}(t)\right)\right]-\frac{\theta_{j}(t)}{k_{i}(t) \theta_{i}(t)+\sum_{j \neq i} k_{j}(t) \theta_{j}(t)}
\end{gathered}
$$


to be considered with the transversality condition $\lim _{t \rightarrow \infty} e^{-\rho t} \lambda_{i i}(t) k_{i}(t)=0$.

Assume now the symmetry conditions $k_{i}(t)=k_{j}(t)=k(t), \theta_{i}(t)=$ $\theta_{j}(t)=\theta(t)$; as a consequence $\lambda_{i i}(t)=\lambda_{j j}(t)=\lambda(t)$. The first order conditions rewrite as follows:

$$
\begin{gathered}
c(t)=1 / \lambda(t) \\
\theta(t)=1 /[N A \lambda(t) k(t)] \Rightarrow c(t)=N k(t) A \theta(t) \\
\dot{\lambda}(t) \cdot[N k(t) \theta(t)]=\lambda(t)\left[\rho-A\left(1-\theta_{i}(t)\right)\right] \cdot[N k(t) \theta(t)]-\theta(t) .
\end{gathered}
$$

Condition (83) may be written as

$$
k(t) \dot{\lambda}(t)=k(t) \lambda(t)\left[\rho-A\left(1-\theta_{i}(t)\right)\right]-(1 / N) .
$$

Moreover, condition (81) may be inserted into the dynamic constraint, to yield:

$$
\lambda(t) \dot{k}(t)=k(t) \lambda(t)[A(1-\theta(t))]-1 .
$$

Summing up (84) and (85) we have:

$$
k(t) \dot{\lambda}(t)+\lambda(t) \dot{k}(t)=\frac{d(k(t) \lambda(t))}{d t}=k(t) \lambda(t) \rho-\frac{1+N}{N}
$$

whose solution is

$$
k(t) \lambda(t)=\frac{(1+N) / N}{\rho}+Q \cdot e^{\rho t}
$$

where $Q$ is an integration constant, which has to be set equal to zero in order to fulfill the transversality condition. Thus,

$$
k(t) \lambda(t)=\frac{(1+N)}{N \rho}
$$

and hence, from (81)

$$
c(t)=\frac{N}{1+N} \rho k(t)
$$

Finally, from (82), we derive

$$
\theta(t)=\frac{1}{A N(\lambda(t) k(t))}=\frac{\rho}{A(1+N)}
$$


By simple substitutions into the state dynamics, we also obtain

$$
\dot{k}(t)=A(1-\theta(t)) k(t)-c(t)=(A-\rho) k(t)
$$

whose solution is

$$
k(t)=k_{0} \exp [(A-\rho) t] .
$$

Thus,

$$
c(t)=\rho \frac{N}{1+N} k_{0} \exp [(A-\rho) t] .
$$

Also in this case, it is immediate to see that consumption and capital increase over time as long as $A>\rho$. To summarise this discussion, we may state:

Proposition 2 The open-loop solution of the Nash game among agents choosing both consumption and taxation is subgame perfect.

\section{A comparative assessment across regimes}

Now we are in a position to compare the relevant variables across three different institutional settings: (i) the Stackelberg game in which the government leads and private agents follow (labelled by the superscript $S E$ ), (ii) the command optimum (labelled by $C O$ ), and (iii) the decentralized equilibrium with no government action (labelled by $D E$ ). The comparison can be performed in the general case that $N$ private agents populate the economy, as well as in the particular case in which $N=1$. We are particularly interested in the performance of the welfare indicator across the aforementioned settings.

The obvious candidate as a measure of welfare is:

$$
V=\int_{0}^{\infty} e^{-\rho t}\{\ln c(t)+\ln G(t)\} d t
$$

describing the total welfare enjoyed by any single private agent in the economy over the time horizon covered by the model. 
In the Stackelberg game, we have

$$
V^{S E}=\int_{0}^{\infty} e^{-\rho t}\left\{\ln \left[\rho k_{0} \cdot e^{\left(\frac{2 A-3 \rho}{2} \cdot t\right)}\right]+\ln \left[\frac{\rho N}{2} k_{0} \cdot e^{\left(\frac{2 A-3 \rho}{2} \cdot t\right)}\right]\right\} d t
$$

which turns out to give:

$$
\begin{aligned}
V^{S E} & =\left[e^{-\rho t} \cdot\left\{-\frac{\ln \left[\rho N k_{0} / 2\right]}{\rho}-\frac{\ln \left[\rho k_{0}\right]}{\rho}-\frac{(2 A-3 \rho)(\rho t+1)}{\rho^{2}}\right\}\right]_{0}^{+\infty}= \\
& =\frac{\ln (1 / 2)+\ln N+2 \ln \left(\rho k_{0}\right)}{\rho}+\frac{(2 A-3 \rho)}{\rho^{2}}
\end{aligned}
$$

Under the command optimum, we have

$$
V^{C O}=\int_{0}^{\infty} e^{-\rho t}\left\{\ln \left[\frac{\rho k_{0}}{2} \cdot e^{(A-\rho) t}\right]+\ln \left[\frac{\rho N}{2} k_{0} \cdot e^{(A-\rho) t}\right]\right\} d t
$$

which amounts to:

$$
\begin{aligned}
V^{C O} & =\left[e^{-\rho t} \cdot\left\{-\frac{\ln \left[\rho N k_{0} / 2\right]}{\rho}-\frac{\ln \left[\rho k_{0}\right]}{\rho}-\frac{2(A-\rho)(\rho t+1)}{\rho^{2}}\right\}\right]_{0}^{+\infty}= \\
& =\frac{\ln (1 / 4)+\ln N+2 \ln \left(\rho k_{0}\right)}{\rho}+\frac{2(A-\rho)}{\rho^{2}}
\end{aligned}
$$

Finally, in the decentralized setting,we have:

$$
V^{D E}=\int_{0}^{\infty} e^{-\rho t}\left\{\ln \left[\frac{\rho N}{1+N} k_{0} e^{[(A-\rho) t]}\right]+\ln \left[\frac{\rho N}{1+N} k_{0} e^{[(A-\rho) t]}\right]\right\}
$$

which is equal to:

$$
\begin{aligned}
V^{D E} & =\left[e^{-\rho t} \cdot\left\{-\frac{2 \ln \left[\rho N k_{0} /(N+1)\right]}{\rho}-\frac{2(A-\rho)(\rho t+1)}{\rho^{2}}\right\}\right]_{0}^{+\infty}= \\
& =\frac{2 \ln [1 /(N+1)]+2 \ln N+2 \ln \left(\rho k_{0}\right)}{\rho}+\frac{2(A-\rho)}{\rho^{2}}
\end{aligned}
$$

It is immediate to check that:

(i) $V^{C O}-V^{S E}=(1-\ln 2) / \rho>0$ for all $N \geq 1$;

(ii) $V^{C O}-V^{D E}=\ln \left[(1+N)^{2} /(4 N)\right] / \rho$, which is nil for $N=1$ and is strictly positive for $N>1$; 
(iii) $V^{S E}-V^{D E}=(1 / \rho)\left(\ln \left[(1+N)^{2} /(2 N)\right]-1\right]$ which is negative for $N=1$ and positive for $N>1$.

All the above results can be summarized as follows:

Proposition 3 (a) if $N>1$, then $V^{C O}>V^{S E}>V^{D E}$; (b) if $N=1$, then $V^{C O}=V^{D E}>V^{S E}$.

In words, if we consider the normalization of population to one, then the decentralised equilibrium and the social optimum coincide, and the policy maker may not be able to improve upon the private optimum by appropriately choosing the tax rate $\theta(t)$. More specifically, if the policy maker acts as a Stackelberg leader in this framework, he leads the system to an inefficient outcome. This is due to the fact that the action of the policy maker introduces strategic interaction in the problem, which - in the absence of an active economic policy by the government - is indeed a simple optimal control problem with a single agent. In sum, normalising the population to one leaves no room for policy making, because the usual prisoners' dilemma associated to the provision of public good disappears and the private provision of public goods is not problematic any more.

As a remedy, to revive active policy making, we propose an alternative framework that duly accounts for the size of the population and the strategic interaction among agents, while preserving the normalisation of the production function. In such a case, the action of the policy maker as the leader permits the economy to reach a better result as compared with the decentralized economy. Obviously, the Stackelberg outcome is inefficient with respect to the command optimum allocation, where strategic interaction among government and private agents is absent by definition. 


\section{Concluding remarks}

This paper has dealt with a well-known issue in the literature on macroeconomic policy, namely the time (in)consistency of the optimal fiscal policy in a dynamic setting where the government acts as a leader and private agents follow. It is well known that, in such a framework, fiscal policy can be time consistent only under specific circumstances. The present paper has focussed on one such circumstance, namely the set of conditions considered in the model recently proposed by Karp and Lee (2003), on the basis of a previous model by Xie (1997). Thanks to specific assumption concerning preferences, they have highlighted a case where optimal fiscal policy is time consistent.

In the present paper we have stressed two features that were not pinpointed by either Xie (1997) or Karp and Lee (2003): (i) under the assumption of logarithmic utility for the representative consumer, the optimal taxation associated with the open-loop Stackelberg outcome is not only time consistent but also subgame perfect because additive separability of the objective function entails that there are no feedback effects, and therefore the resulting optimal open-loop policy is stationary; (ii) the normalization of the population of consumers to one (which is a standard assumption in this line of literature, and usually accompanies all those analyses based on the representative agent hypothesis) indeed plays a significant role in shaping the properties of the fiscal policy: specifically, it rules out the problems connected with the inefficiency of the private provision of public good, and, in so doing, it also eliminates the scope for active policy-making.

Our model has shown that two different sources of inefficiency operate in this model, when a multiplicity of private agents is duly accounted for. The first is represented by the "standard" inefficiency usually associated with the private provision of public goods, which disappears if the "game" collapses 
into an optimal control model when the size of the population is normalised to unity. The second type of inefficiency is entailed by the interaction between the private sector and the government, which arises, on the contrary, if the population is indeed normalised to one. Under this assumption, the performance of the decentralised economy would exactly replicate the command optimum; as a consequence, transforming the optimal control problem of the single agent into a Stackelberg game (whereby strategic interaction is introduced ad hoc in a model that a priori is not strategic) yields an inefficient outcome.

Moreover, we have shown that the subgame perfection (or strong time consistency) of the optimal fiscal rules produced by the model are robust to the introduction of proper strategic interaction among private agents. This has also allowed us to proceed to a thorough assessment of the welfare properties of the decentralised economy, the command optimum and the Stackelberg game. In particular, our core result is that there is a scope for policy making, with the government taking the leader's role, only if the economy consists of at least two agents. If so, then intuitively the decentralised equilibrium is the outcome of a prisoners' dilemma with welfare falling short of the first best. 


\section{References}

[1] Alvarez, F., P.J. Kehoe and P.A. Neumeyer (2004), "The Time Consistency of Optimal Monetary and Fiscal Policies", Econometrica, 72, 541-67.

[2] Barro, R.J. and D.B. Gordon (1983a), "A Positive Theory of Monetary Policy in a Natural Rate Model", Journal of Political Economy, 91, 589-610.

[3] Barro, R.J. and D.B. Gordon (1983b), "Rules, Discretion and Reputation in a Model of Monetary Policy", Journal of Monetary Economics, 12, 101-21.

[4] Calvo, G. (1978), "On the Time Consistency of Optimal Policy in a Monetary Economy", Econometrica, 46, 1411-28.

[5] Cellini, R., L. Lambertini and G. Leitmann (2004), "Perfect Uncontrollable Differential Games", working paper no. 509, Dipartimento di Scienze Economiche, Università degli Studi di Bologna.

[6] Cohen, D. and P. Michel (1988), "How Should Control Theory Be Used to Calculate a Time-Consistent government Policy?", Review of Economic Studies, 55, 263-74.

[7] Dockner, E.J, S. Jørgensen, N.V. Long and G. Sorger (2000), Differential Games in Economics and Management Science, Cambridge, Cambridge University Press.

[8] Karp, L. and I.H. Lee (2003), Time-Consistent Policies, Journal of Economic Theory, 112, 353-64.

[9] Kydland, F. (1977), "Equilibrium Solutions in Dynamic DominantPlayer Models", Journal of Economic Theory, 15, 307-24. 
[10] Kydland, F. and E. Prescott (1977), "Rules rather than Discretion: The Inconsistency of Optimal Plans", Journal of Political Economy, 85, 47392.

[11] Lucas, R.E. Jr. and N.L. Stokey (1983), "Fiscal and Monetary Policy in an Economy without Capital", Journal of Monetary Economics, 12, $55-93$.

[12] Persson, T. and G. Tabellini (1990), Macroeconomic Policy, Credibility and Politics, Chur, Harwood Academic Publishers.

[13] Persson, T. and G. Tabellini (2000), Political Economics. Explaining Economic Policy, Cambridge, MA, MIT Press.

[14] Simaan, M. and J.B. Cruz, Jr. (1973a), "On the Stackelberg Strategy in Nonzero Sum Games", Journal of Optimization Theory and Applications, 11, 533-55.

[15] Simaan, M. and J.B. Cruz, Jr. (1973b), "Additional Aspects of the Stackelberg Strategy in Nonzero Sum Games", Journal of Optimization Theory and Applications, 11, 613-26.

[16] Xie, D. (1997), "On Time Consistency: A Technical Issue in Stackelberg Differential Games", Journal of Economic Theory, 76, 412-30. 